\title{
Dynamics and spatio-temporal distribution of geomagnetic disturbances during periods of increased solar activity and magnetic storms
}

\author{
Oksana Mandrikova ${ }^{1, *}$, Anastasia Rodomanskay ${ }^{1}$ \\ ${ }^{1}$ Institute of Cosmophysical Research and Radio Wave Propagation FEB RAS, 684034 Paratunka, \\ Kamchatka region, Russia
}

\begin{abstract}
A detailed spatio-temporal analysis of magnetic data was performed during the periods of magnetic storms on October 02, 2013 and September 27, 2019 based on measurements of the station network. In this work, we used a method developed by us for the analysis of magnetic data, based on the use of wavelet transform and adaptive thresholds. The method allows us to identify short-period field disturbances and estimate their intensity from the data of the H-component of the geomagnetic field. The features of the occurrence and propagation of geomagnetic disturbances in the auroral zone and at meridionally located stations have been studied. Dynamic spectra of disturbances of different intensity and duration are obtained. The paper confirms the possibility of occurrence of short-period weak geomagnetic disturbances at stations from high latitudes to the equator, preceding magnetic storms and correlating with fluctuations of the southern Bz-component of the interplanetary magnetic field and increases in the auroral indices of geomagnetic activity. Cross-correlation dependences of the intensity of geomagnetic disturbances on the parameters of the interplanetary medium during magnetic storms were obtained from the data of the network of magnetic stations. A statistically significant influence of the magnitude of the scope of the Bz-component of the IMF and the speed of the solar wind on the development of magnetic storms during the initial and main phases of magnetic storms was revealed.
\end{abstract}

\section{Introduction}

The analysis of time series of geophysical parameters is an important basis for methods of studying the processes occurring in near-earth space during periods of increased solar activity and magnetic storms. The article pays special attention to the development of methods for analyzing the geomagnetic signal, which characterizes the complex space-time structure and dynamics of the variable geomagnetic field. A detailed characterization of fluctuation phenomena in geomagnetic signals at various scales from global effects to local disturbances is important for understanding the intensity, type and development of a magnetic storm. The problem of processing and analyzing direct experimental data is related to their complex irregular structure, the presence of features of different shapes, amplitudes and

${ }^{*}$ Corresponding author: oksanam1@mail.ru 
durations [1-6]. Isolating and identifying these features is a complex task. Traditional approaches mainly use basic methods of time series analysis, which include various smoothing operations, provide a rather general picture, lead to smoothing of local disturbances, which often contain important information about the conditions of the geomagnetic field and are clearly associated with the development of magnetic storms.

The paper proposes a method for analyzing geomagnetic data based on wavelet transform to overcome the above limitations. Wavelet transform is one of the most effective methods for analyzing data of complex structure [7-8]. On the basis of the wavelet transform, an automated method for detecting geomagnetic pulsations has been developed [9-10], an algorithm has been proposed for automatic detection of magnetic storms with an increased risk of the formation of geomagnetically-induced currents [11], a method for calculating the geomagnetic activity index WISA [3], etc.

The article presents the results of processing and analysis of data from a network of magnetic stations in Russia («Yakutsk» YAK, «Paratunka» PET, http://www.intermagnet.org), equatorial stations («Guam» GUA, USA, http: // www. intermagnet.org) and the network of stations in the auroral zone («Abisko» ABK, Sweden; «Barrow» BRW, USA; «Narsarsuaq» NAQ, Greenland; «Yellowknife» YKC, Canada, http: //www.intermagnet.org). The authors carried out a cross-correlation study between the measure of the intensity of geomagnetic disturbances obtained during the application of the data analysis technique developed on the basis of the wavelet transform [12] and the data of the interplanetary medium (https://omniweb.gsfc.nasa.gov/ow.html ). Cross-correlation analysis is a widely used tool for studying the relationship between data from the interplanetary medium and the magnetosphere [13-14]. The results obtained confirmed the uniqueness and effectiveness of the proposed method, and also showed the possibility of its application for assessing and monitoring the state of the geomagnetic field in various magnetic observatories without a specific adaptation to their location.

\section{Description of the method}

Data analysis was performed based on the wavelet transform [15-16]. The construction of continuous wavelet transform (CWT) data was applied:

$$
\left(W_{\psi} f\right)(b, a):=|a|^{-1 / 2} \int_{-\infty}^{\infty} f(t) \Psi\left(\frac{t-b}{a}\right) d t, \psi-\text { wavelet } f \in L^{2}(R), a, b \in R, a \neq 0 \text {. }
$$

The amplitude of the wavelet coefficients characterizes the amplitude of the local singularity of the function $f$ in the vicinity of the point $t=b$ on the scale $a$. Therefore, in the analysis of time series, an increase in the amplitude of the wavelet coefficients indicates the occurrence of an anomaly in the data in the vicinity of the point $t=b$. Then the amplitude of the wavelet coefficients:

$$
v_{b, a}=\left|\left(W_{\psi} f\right)(b, a)\right|
$$

can be taken as a measure of the intensity of geomagnetic disturbances on a scale $a$ at a point in time $t=b$.

In this case, the intensity of disturbances at a time $t=b$ can be estimated based on the value:

$$
I_{b}=\sum_{a}\left|\left(W_{\psi} f\right)(b, a)\right|
$$

The use of operations (1) and (2) makes it possible to identify and evaluate anomalous changes in the dynamics of field variations that characterize the degree of its disturbance at the observation site. 
As an example, fig. 1 shows the results of applying operations (1) and (2) to the geomagnetic field data of the Yakutsk, Paratunka and Guam stations during the geomagnetic storm on October 2, 2013, caused by a coronal mass ejection (http: / /spaceweather.com). The estimates obtained have minute time resolution, which allows obtaining detailed operational information about the state of the geomagnetic field. Before the analyzed magnetic storm, the speed of the solar wind did not exceed $350 \mathrm{~km} / \mathrm{s}$. the Bz-component of the interplanetary magnetic field (IMF) varied in the range of $+/-5 \mathrm{nT}$. The spectral-temporal structure of disturbances (fig. 1g), both on the eve and during the event, had the same character at the analyzed stations, which is probably due to the influence of the global ring current. Before the onset of a geomagnetic storm, one can observe periods of a moderate increase in geomagnetic activity, which correlate with the periods of southward turns of the Bz-component IMF and a moderate increase in the indices of auroral activity (fig. $2 \mathrm{a}$ and $2 \mathrm{~g}$, h, October 01 from 13:30 to 16:00 UT; October 01 from 21:00 to 23:30 UT). Analysis of field variations (Figs. $1 \mathrm{~g}$, $\mathrm{h}$ and $2 \mathrm{~g}, \mathrm{~h}$ ) shows the presence of anomalous changes in the data during this period, which confirms the effectiveness of using operation (1) and (2) for detecting changes in variations in the geomagnetic field, including small amplitude. At the beginning of the day on October 02 (from 02:00 UT), the solar wind speed began to increase, fluctuations of the Bz-component IMF increased, and geomagnetic disturbances arose at the analyzed stations.

Taking into account the nonstationarity of the analyzed data for the identification of shortperiod anomalies, the adaptive thresholds were used:

$$
F_{T_{a}}\left[\left(W_{\psi} f\right)(b, a)\right]=\left\{\begin{array}{l}
\left(W_{\psi} f\right)(b, a), \text { if }\left(W_{\psi} f\right)(b, a) \geq T_{a} \\
0, \text { if }\left|\left(W_{\psi} f\right)(b, a)\right|<T_{a} \\
-\left(W_{\psi} f\right)(b, a), \text { if }\left(W_{\psi} f\right)(b, a) \leq-T_{a}
\end{array}\right.
$$

where $T_{a}=U \times S t_{a}$ is the threshold function, $S t^{l}{ }_{a}=\sqrt{\frac{1}{l-1} \sum_{k=1}^{l}\left(\left(W_{\psi} f\right)(b, a)-\overline{\left(W_{\psi} f\right)(b, a)}\right)^{2}}$ $\overline{\left(W_{\psi} f\right)(b, a)}$ is the average value of the coefficients of the continuous wavelet transform, calculated in a sliding time window of length $l, U$ is the threshold coefficient. The length of the sliding time window determines the size of the window within which we estimate the disturbances. The intensity of short-period positive and negative disturbances at a time $t=b$ was determined based on the value:

$$
E_{b}^{ \pm}=\sum_{a} F_{T_{a}}\left[\left(W_{\psi} f\right)(b, a)^{ \pm}\right] .
$$

In fig. 3 and 4 show the results of applying operations (3) and (4) with the following parameters: coefficient $U=2$ and length of the sampling window $l=720$ (corresponds to 12 hours). An analysis of the results presented in fig. $3 \mathrm{e}, \mathrm{f}$ and $4 \mathrm{e}, \mathrm{f}$ confirms the efficiency of operation (3), the use of which makes it possible to single out nonstationary short-period changes in the data characterizing the occurrence of weak increases in geomagnetic activity that preceded a major magnetic event. The identified disturbances appeared synchronously at stations from high latitudes to the equator (fig. $3 \mathrm{e}, \mathrm{f}$ and $4 \mathrm{e}, \mathrm{f}$ ), correlated with an increase in auroral indices (fig.3a and 4a), which indicates their relationship with short-term (instantaneous) changes in the parameters of the interplanetary medium [17-18]. In the initial phase of the storm, the intensity of geomagnetic disturbances at the analyzed stations increased sharply (see fig. 3e, f and 4e, f). The use of operations (3) and (4) made it possible to identify and evaluate nonstationary short-period increases in geomagnetic activity, which gives a more detailed and deep understanding of the dynamics of geomagnetic processes. 
a)

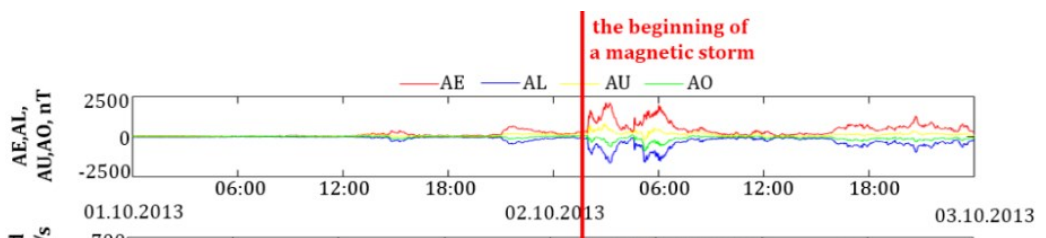

b)

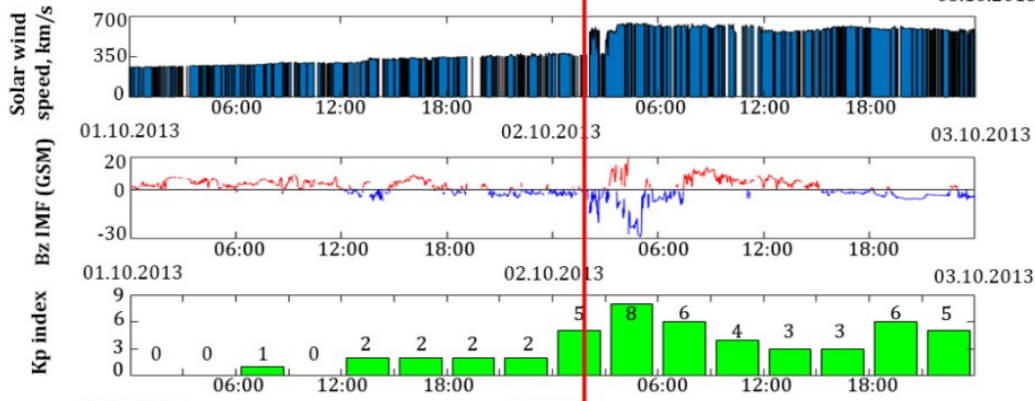

e)

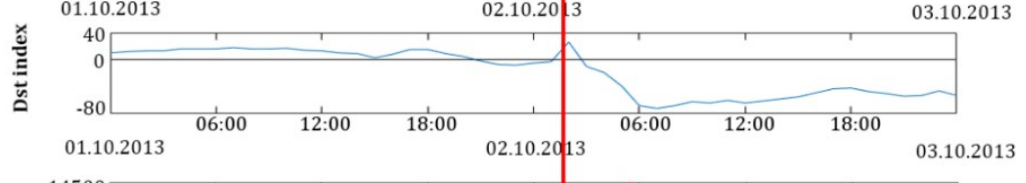

f)

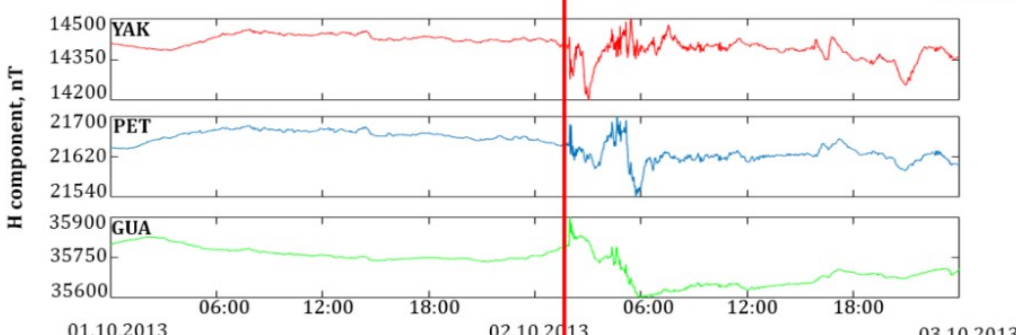

g)

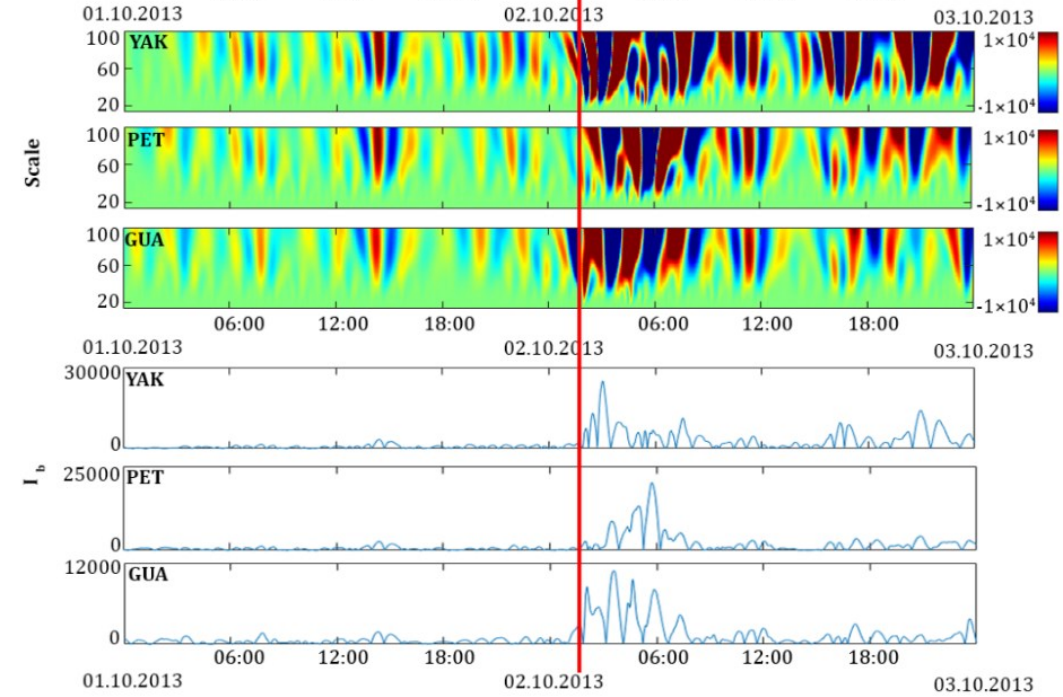

Fig. 1. Results of data processing for the period from 01.10.2013 to 03.10.2013:

a) auroral indices $\mathrm{AE}, \mathrm{AO}, \mathrm{AL}$ and $\mathrm{AU}$; b) solar wind speed; c) Bz-component of IMF, positive values are shown in red, negative values are shown in blue; d) Kp-index; e) Dst index; f) Hcomponents of the geomagnetic field of PET, YAK and GUA stations; g) wavelet spectrum of geomagnetic disturbances in the areas of PET, YAK and GUA stations (operation (1)); h) absolute values of the intensity of disturbances in the areas of PET, YAK and GUA stations (operation (2)). The vertical line indicates the beginning of the magnetic storm. 
a)

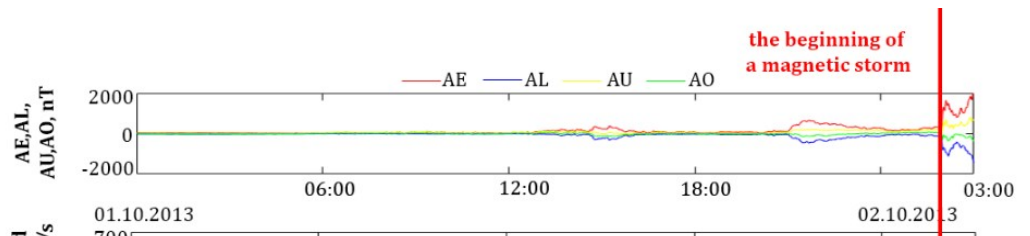

b)

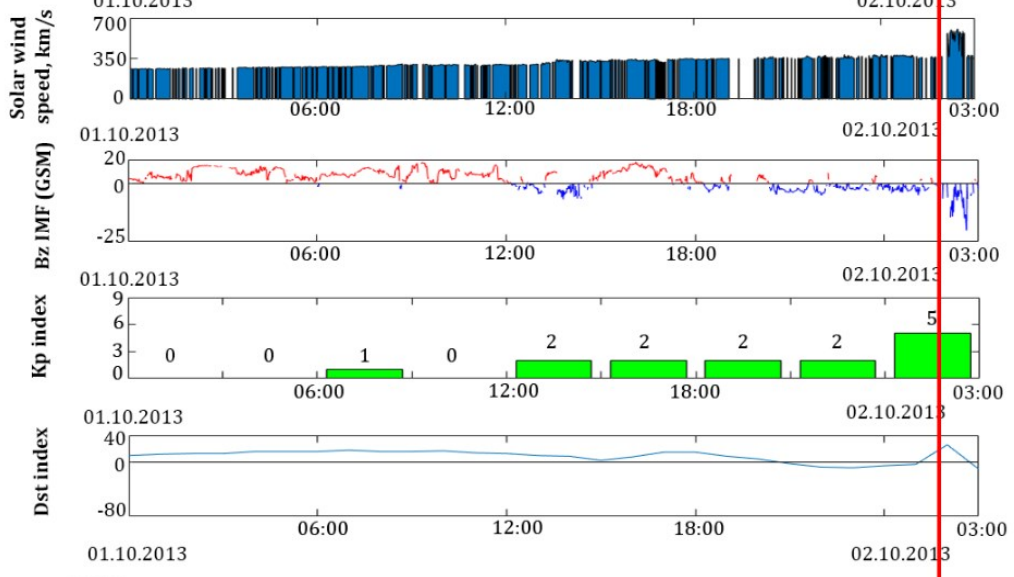

d)

e)

f)

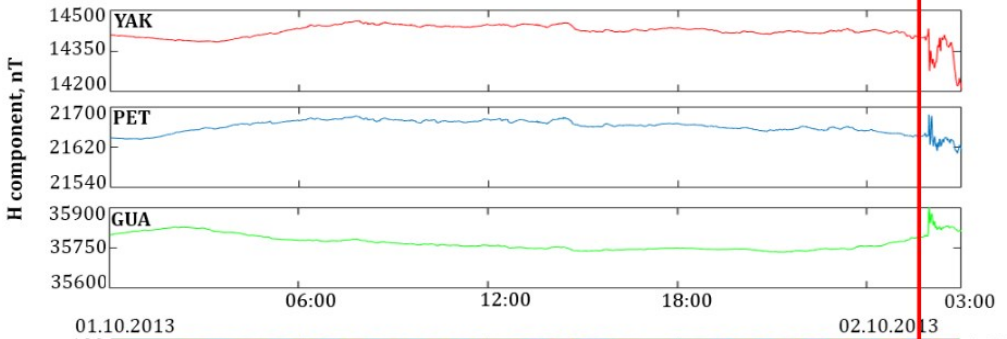

g)

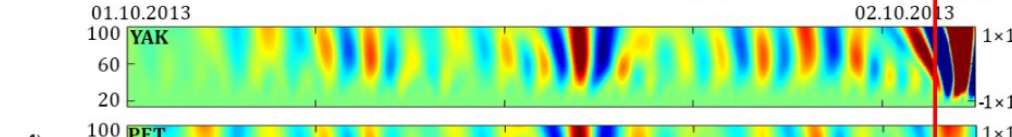

h)

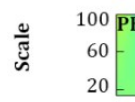
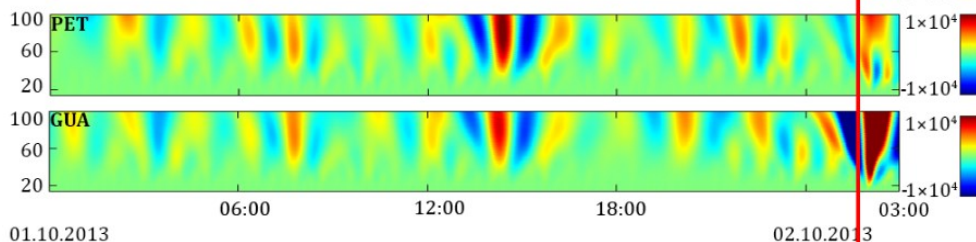

01.10 .2013
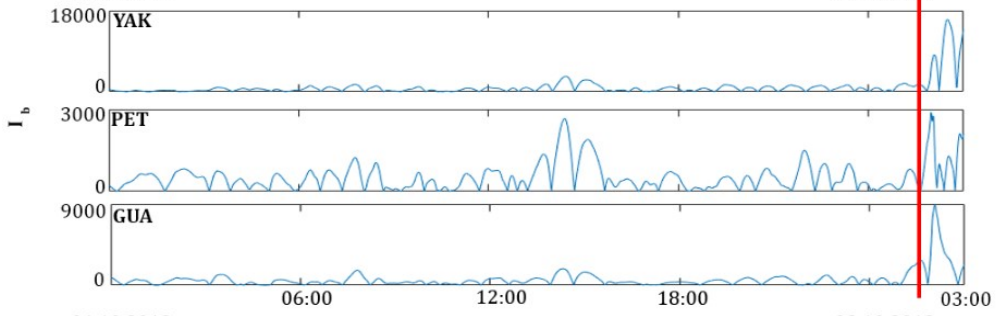

01.10 .2013

02.10 .2013

Fig. 2. Results of data processing before the start of the geomagnetic storm on 02.10.2013: a) auroral indices $\mathrm{AE}, \mathrm{AO}, \mathrm{AL}$ and $\mathrm{AU}$; b) solar wind speed; c) Bz-component of IMF, positive values are shown in red, negative values are shown in blue; d) Kp-index; e) Dst index; f) Hcomponents of the geomagnetic field of PET, YAK and GUA stations; g) wavelet spectrum of geomagnetic disturbances in the areas of PET, YAK and GUA stations (operation (1)); h) absolute values of the intensity of disturbances in the areas of PET, YAK and GUA stations (operation (2)). The vertical line indicates the beginning of the magnetic storm. 
a)

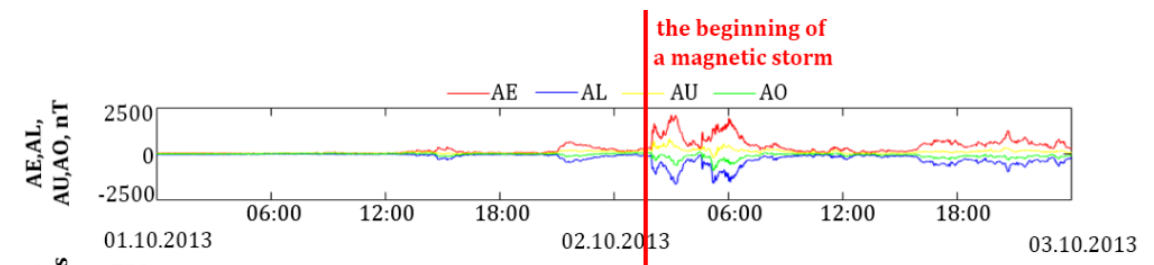

b)

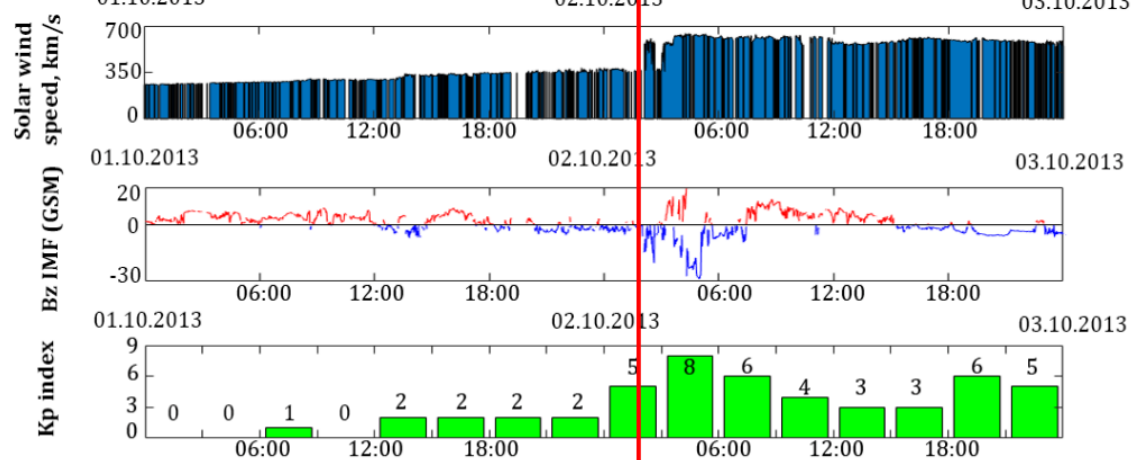

c)

d)

e)

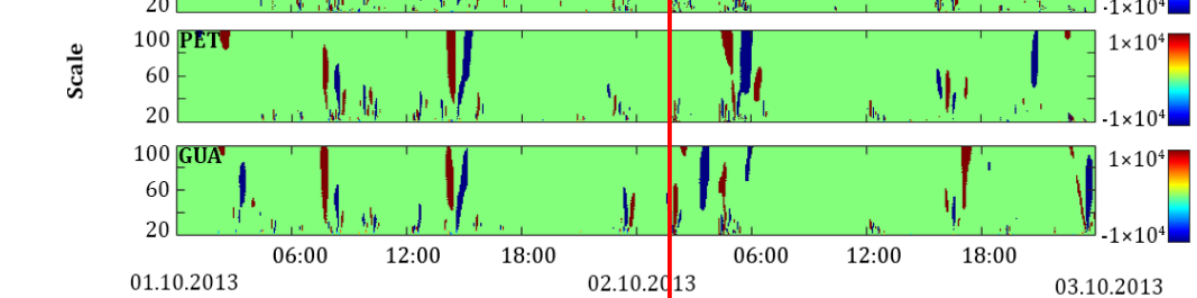

f)

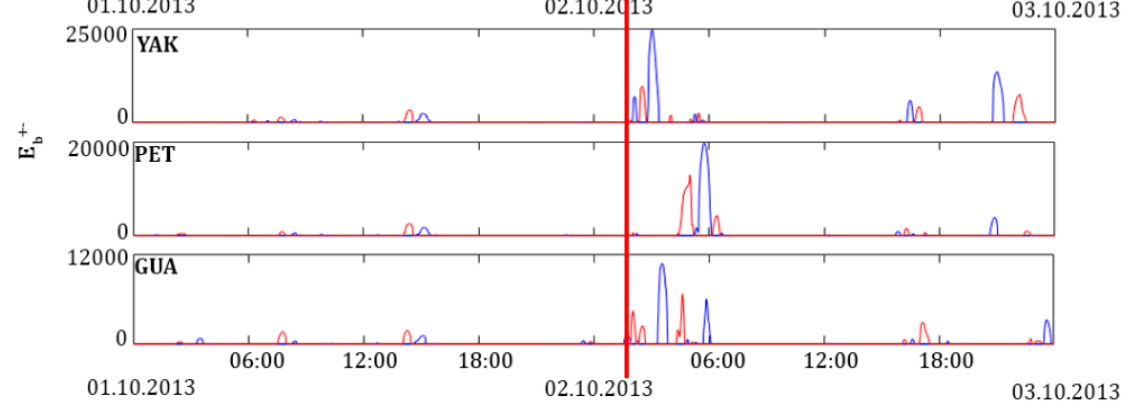

Fig. 3. Results of data processing for the period from 01.10.2013 to 03.10.2013:

a) auroral indices $\mathrm{AE}, \mathrm{AO}, \mathrm{AL}$ and $\mathrm{AU}$; b) solar wind speed; c) Bz-component of IMF, positive values are shown in red, negative values are shown in blue; d) Kp-index; e) positive (red) and negative (blue) disturbances at PET, YAK and GUA stations (operation (3), the sliding time window size is 720 samples); f) intensity of positive (red) and negative (blue) disturbances at PET, YAK and GUA stations (operation (4)). The vertical line indicates the beginning of the magnetic storm. 
a)

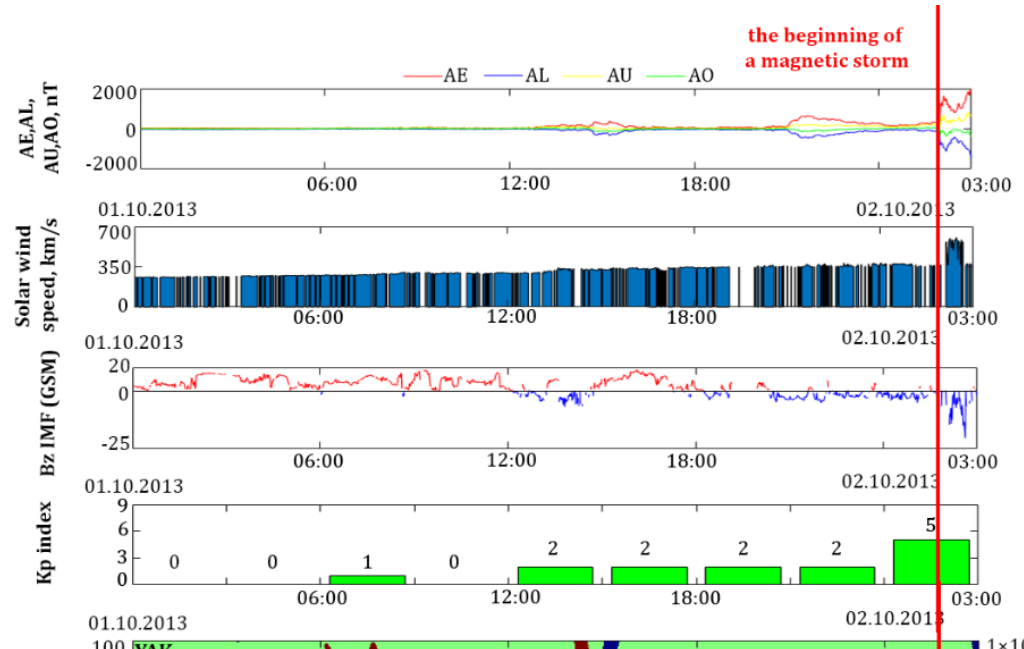

d)

e)

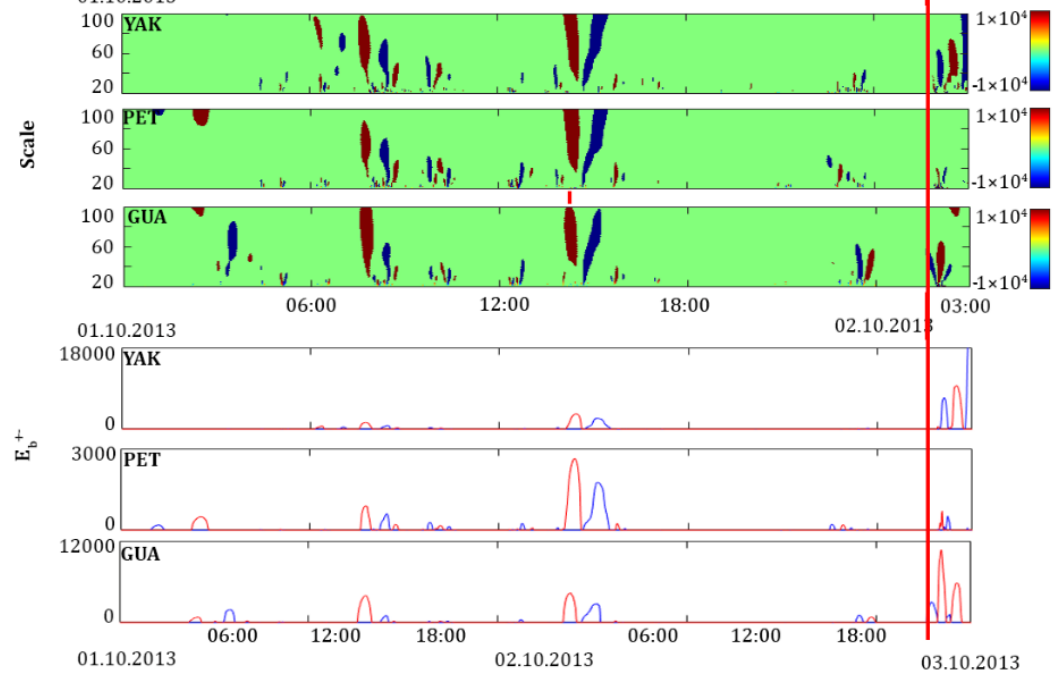

Fig. 4. Results of data processing before the start of the geomagnetic storm on 02.10.2013:

a) auroral indices $\mathrm{AE}, \mathrm{AO}, \mathrm{AL}$ and $\mathrm{AU}$; b) solar wind speed; c) Bz-component of IMF, positive values are shown in red, negative values are shown in blue; d) Kp-index; e) positive (red) and negative (blue) disturbances at PET, YAK and GUA stations (operation (3), the sliding time window size is 720 samples); f) intensity of positive (red) and negative (blue) disturbances at PET, YAK and GUA stations (operation (4)). The vertical line indicates the beginning of the magnetic storm.

\section{Experimental results and discussion}

In this work, the processing of geomagnetic data with minute resolution of the network of magnetic stations of Russia «Yakutsk» YAK, «Paratunka» PET, equatorial stations «Guam» GUA and the network of stations in the auroral zone «Abisko» ABK, Sweden; «Barrow» BRW, USA; «Narsarsuaq» NAQ, Greenland; «Yellowknife» YKC, Canada (Table 1 and Figure 5). We used magnetic data obtained at observatories in accordance with the INTERMAGNET standards (www.inrtermagnet.org), that is, data without noise, jumps and long-term artificial and technogenic effects. The analysis results were compared with IMF data and solar wind parameters (https://omniweb.gsfc.nasa.gov/ow.html). 
Table 1. Observatories the data of which were used

\begin{tabular}{|l|c|c|c|c|c|c|}
\hline \multicolumn{1}{|c|}{ Observatory } & $\begin{array}{c}\text { IAGA } \\
\text { code }\end{array}$ & $\begin{array}{c}\text { Geographical } \\
\text { latitude }\end{array}$ & $\begin{array}{c}\text { Geographical } \\
\text { longitude }\end{array}$ & $\begin{array}{c}\text { Geomagnetic } \\
\text { latitude }\end{array}$ & $\begin{array}{c}\text { Geomagnetic } \\
\text { longitude }\end{array}$ & $\begin{array}{c}\text { Local } \\
\text { time } \\
\text { (LT) }\end{array}$ \\
\hline Barrow & BRW & $71^{0} 19.5^{\prime} \mathrm{N}$ & $156^{0} 37.2^{\prime} \mathrm{W}$ & $70^{0} 06.0^{\prime} \mathrm{N}$ & $110^{0} 34,2 \mathrm{~W}$ & UTC- 8 \\
\hline Yellowknife & YKC & $62^{0} 28.8^{\prime} \mathrm{N}$ & $114^{0} 28.8^{\prime} \mathrm{W}$ & $67^{0} 12.0^{\prime} \mathrm{N}$ & $28^{0} 51,0 \mathrm{~W}$ & UTC-6 \\
\hline Narsarsuaq & NAQ & $61^{0} 11.7^{\prime} \mathrm{N}$ & $45^{0} 25.0^{\prime} \mathrm{W}$ & $69^{0} 00.0^{\prime} \mathrm{N}$ & $38^{0} 49.2^{\prime} \mathrm{W}$ & UTC-2 \\
\hline Abisko & ABK & $68^{0} 21.7^{\prime} \mathrm{N}$ & $18^{0} 43.4^{\prime} \mathrm{E}$ & $66^{0} 04.8^{\prime} \mathrm{N}$ & $113^{0} 53.4^{\prime} \mathrm{E}$ & UTC+2 \\
\hline Yakutsk & YAK & $61^{0} 57.6^{\prime} \mathrm{N}$ & $129^{0} 39.4^{\prime} \mathrm{E}$ & $52^{0} 54.0^{\prime} \mathrm{N}$ & $162^{0} 23.4^{\prime} \mathrm{E}$ & $\mathrm{UTC}+09$ \\
\hline Guam & GUA & $13^{0} 35.4^{\prime} \mathrm{N}$ & $144^{0} 52.5^{\prime} \mathrm{E}$ & $5^{0} 58.8^{\prime} \mathrm{N}$ & $143^{0} 03.0^{\prime} \mathrm{E}$ & UTC+10 \\
\hline Paratunka & PET & $52^{0} 58.3^{\prime} \mathrm{N}$ & $158^{0} 15.0^{\prime} \mathrm{E}$ & $46^{0} 19.8^{\prime} \mathrm{N}$ & $136^{0} 52.2^{\prime} \mathrm{E}$ & UTC+12 \\
\hline
\end{tabular}

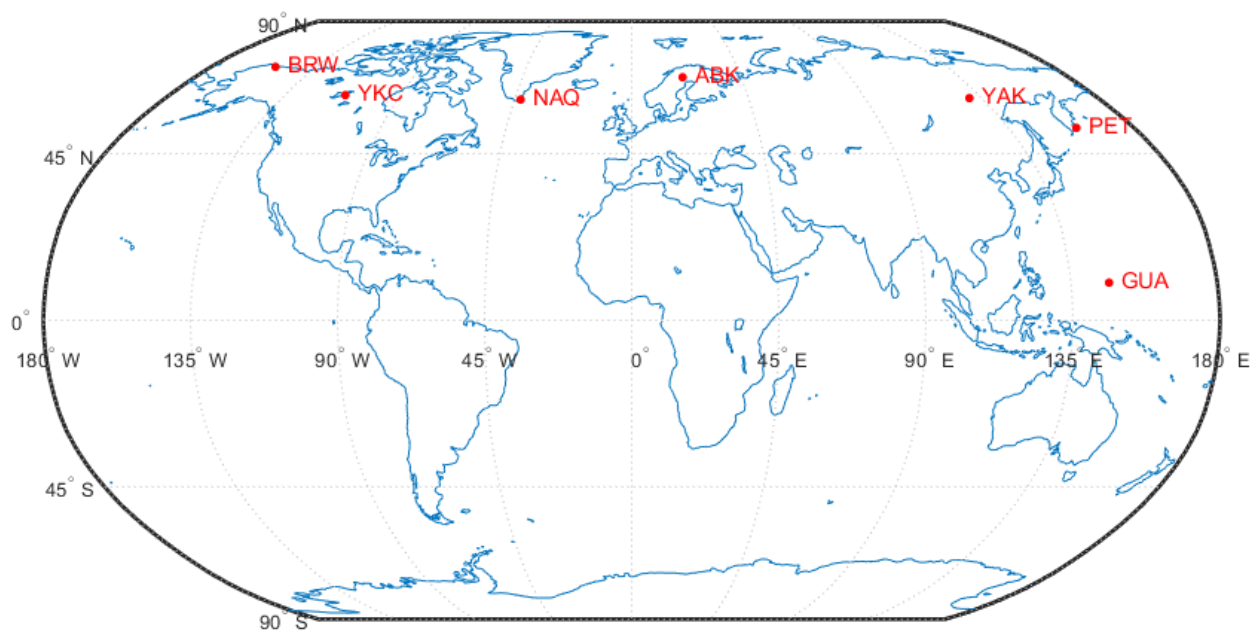

Fig. 1. Geographical position of observatories that provided data used in this study.

The analyzed geomagnetic storm, which occurred on September 27, 2019 (fig. 6-9), was caused by the arrival of a heterogeneous accelerated flow flowing from a coronal hole (http://spaceweather.com). On the eve of the magnetic storm on September 26 from 01:00 to 03:00 UT, during the period of negative values of the Bz-component (fig.6b), pronounced synchronous geomagnetic disturbances occurred at all analyzed stations (fig.6f, g, 7d, 8d, e, and 9d). Possibly, the identified weak field disturbances are a residual phenomenon from the substorm, which was recorded on September 25, 2019 at 22:08 UT (http://supermag.jhuapl.edu). Further, synchronous short-term increases in geomagnetic activity are observed during the southward rotation of the Bz-component IMF at about 15:00 UT at auroral stations (fig. 7d and 9d) and at all meridionally located stations (fig. 8d, e). At the end of the day on September 26 (from 22:00 to 24:00 UT), weak short-term increases in geomagnetic activity occurred at the auroral stations NAQ and ABK (fig.9d), and simultaneously at the equatorial station GUA (fig. 8e). The results obtained indicate the external nature of the identified geomagnetic disturbances and their relationship with the approaching magnetic storm. 
a)

b)
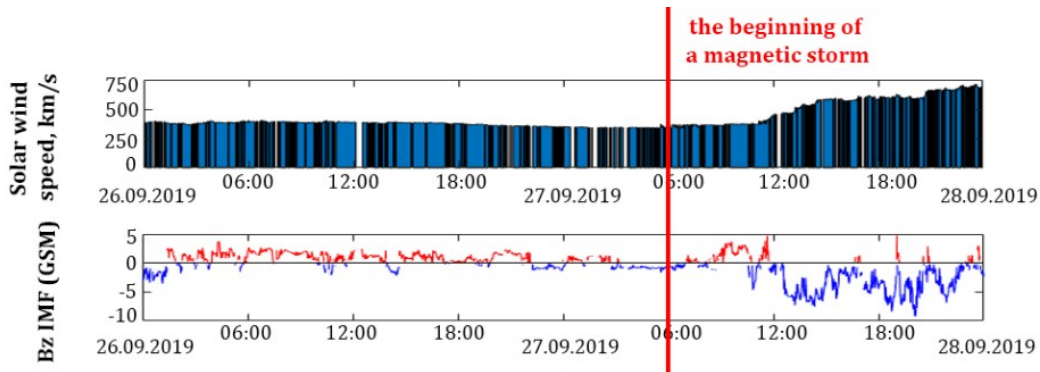

d)

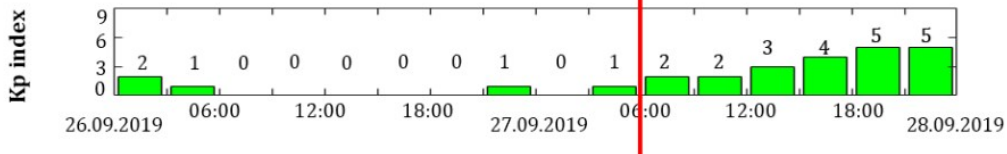

e)

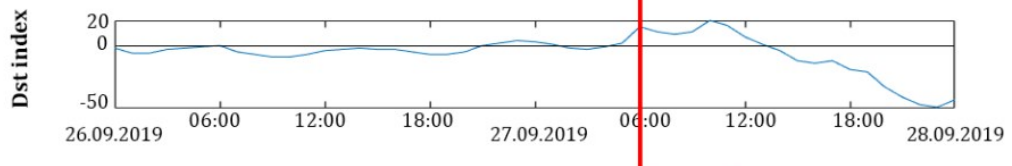

f)

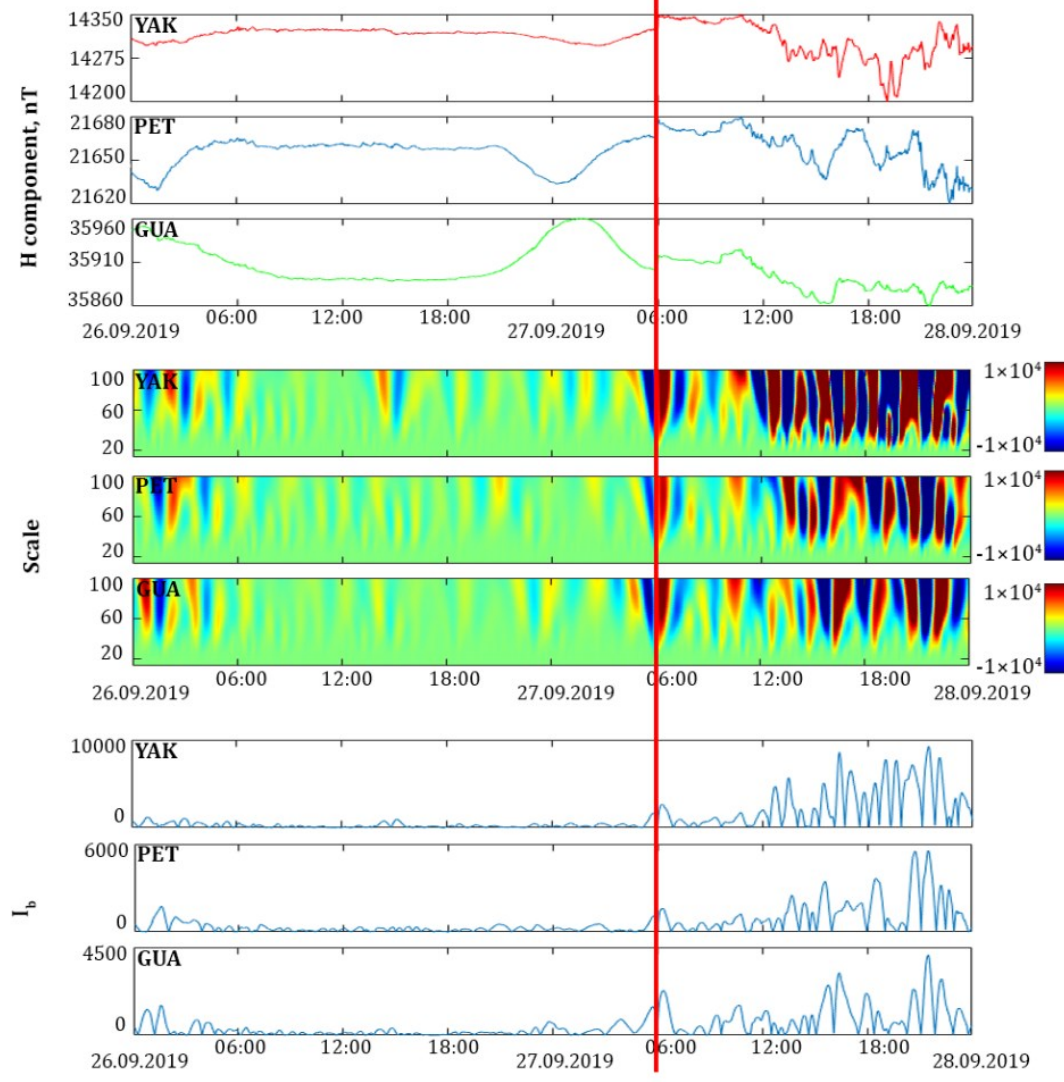

Fig. 6. Results of data processing for the period from 26.09.2019 to 28.09.2019:

a) solar wind speed; b) Bz-component of IMF, positive values are shown in red, negative values are shown in blue; c) Kp-index; d) Dst index; e) H-components of the geomagnetic field of PET, YAK and GUA stations; f) wavelet spectrum of geomagnetic disturbances in the areas of PET, YAK and GUA stations (operation (1)); g) absolute values of the intensity of disturbances in the areas of PET, YAK and GUA stations (operation (2)). The vertical line indicates the beginning of the magnetic storm. 
a)

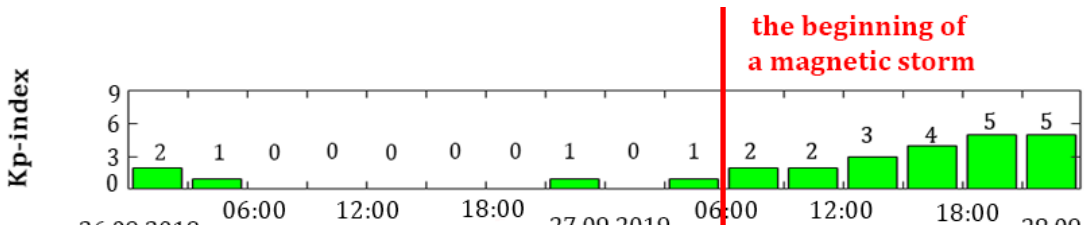

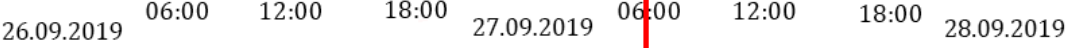

b)

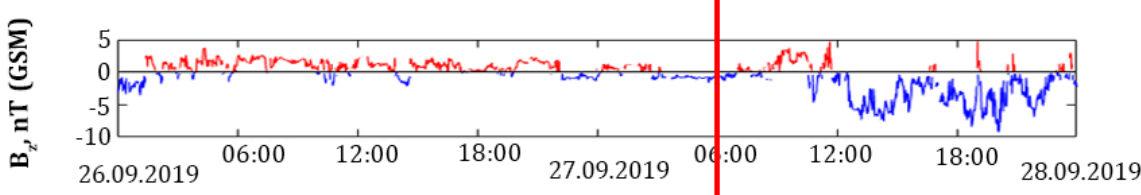

c)

Universal time

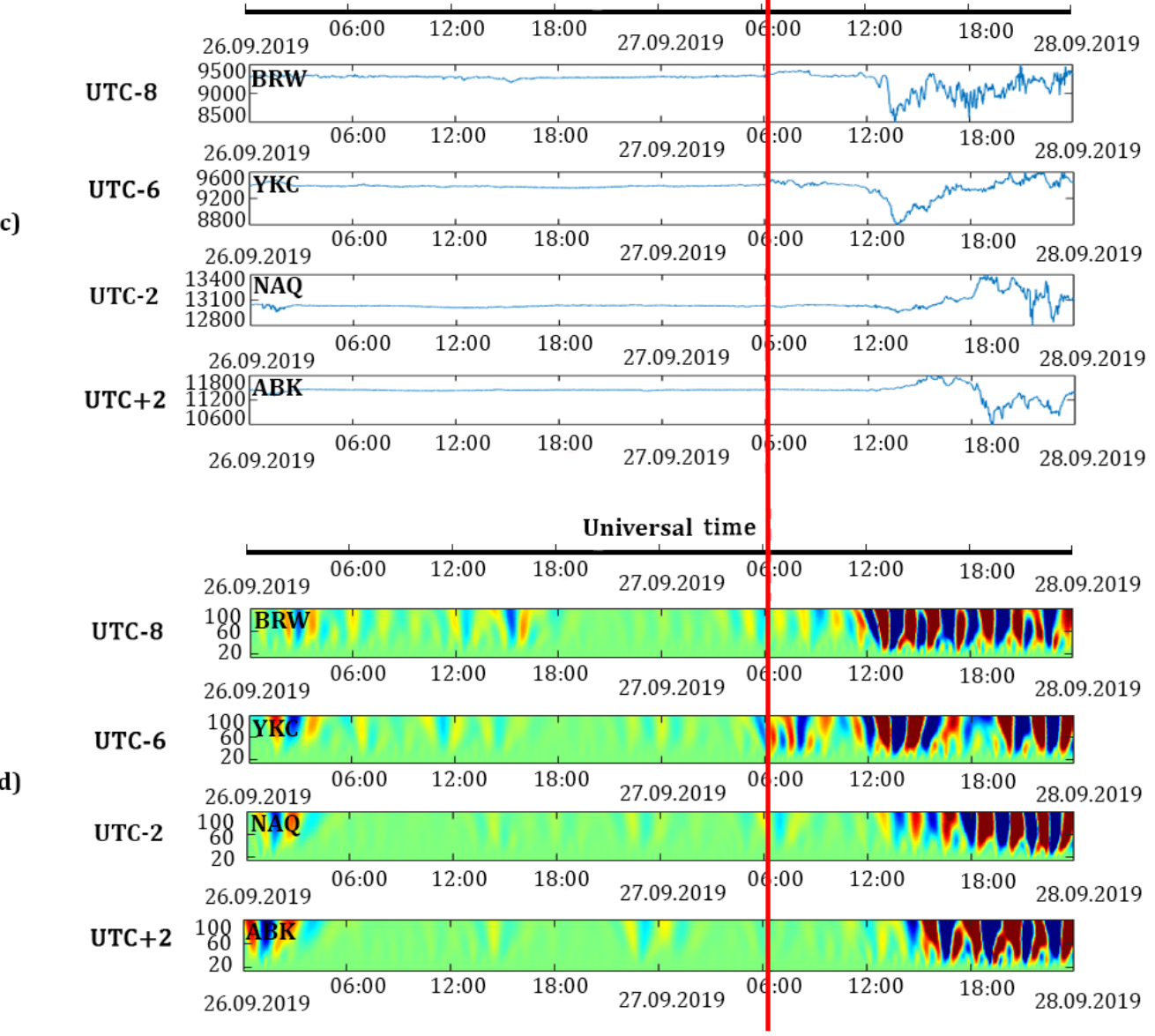

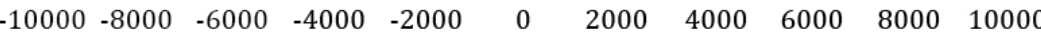

Fig. 7. Results of processing data from auroral stations for the period from 26.09.2019 to 28.09.2019: a) Kp-index; b) Bz-component of IMF, positive values are shown in red, negative values are shown in blue; c) H-components of the geomagnetic field of auroral stations; d) wavelet spectrum of geomagnetic disturbances in the regions of auroral stations (operation (1)). The vertical line indicates the beginning of the magnetic storm. 
a)

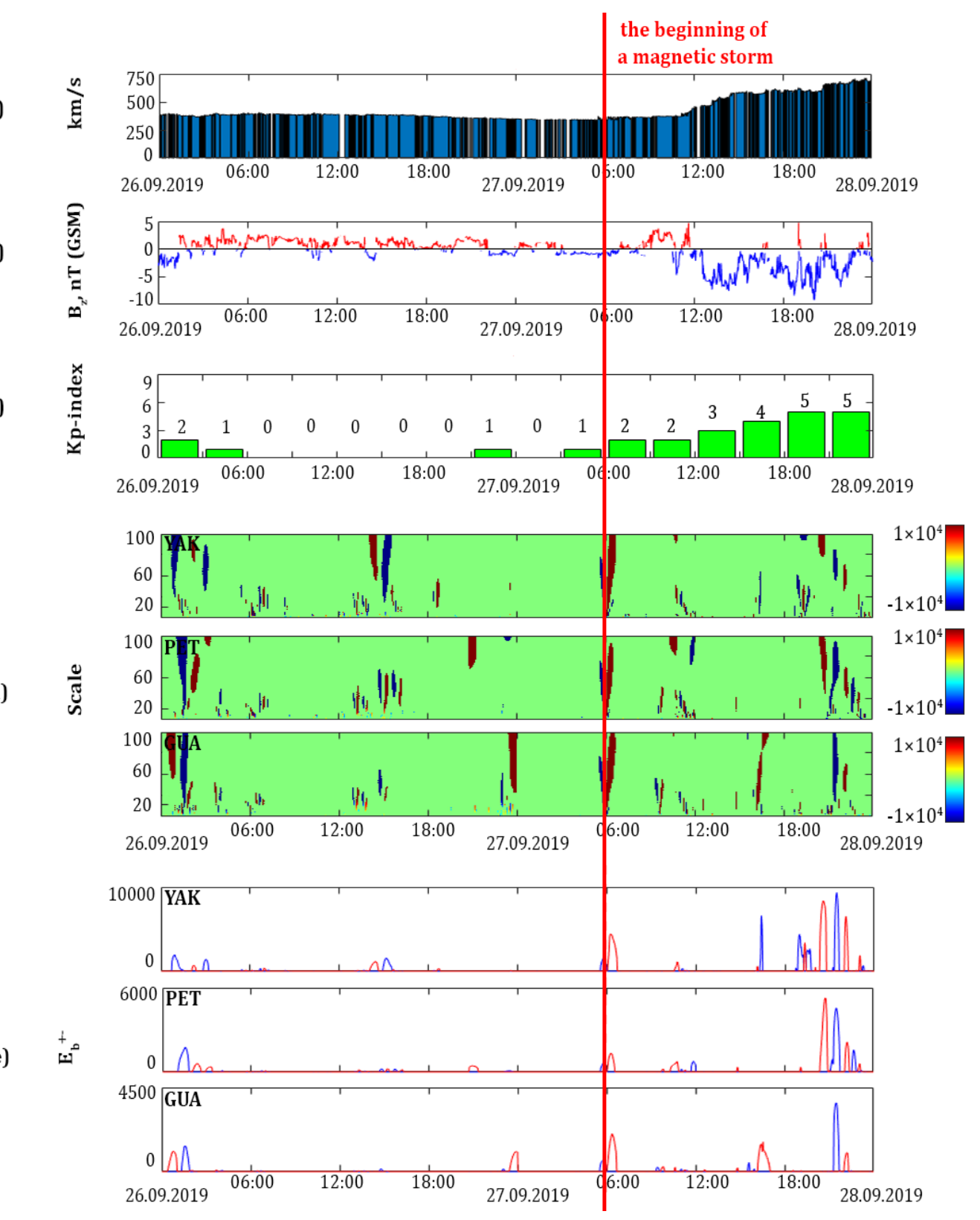

b)

c)

Fig. 8. Results of data processing for the period from 26.09.2019 to 28.09.2019:

a) solar wind speed; b) Bz-component of IMF, positive values are shown in red, negative values are shown in blue; c) Kp-index; d) positive (red) and negative (blue) disturbances at PET, YAK and GUA stations (operation (3), sliding time window size is 720 samples); e) intensity of positive (red) and negative (blue) disturbances at PET, YAK, and GUA stations (operation (4)). The vertical line indicates the beginning of the magnetic storm. 
a)

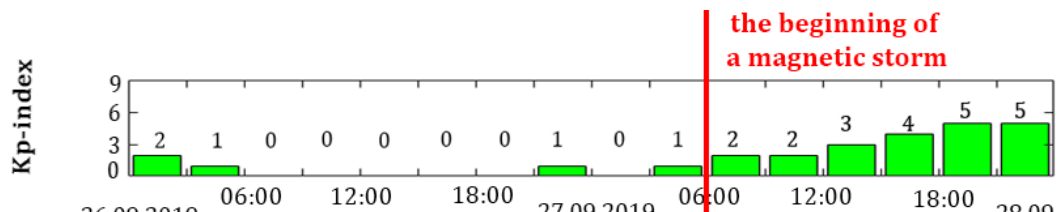
26.09 .2019

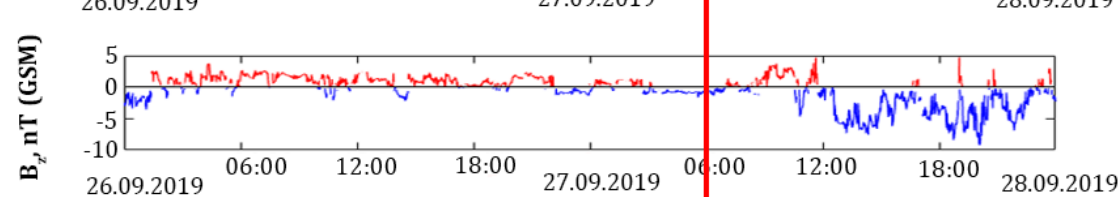

c)

b)

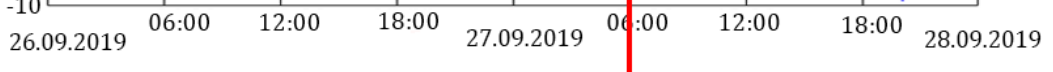

\section{Universal time}

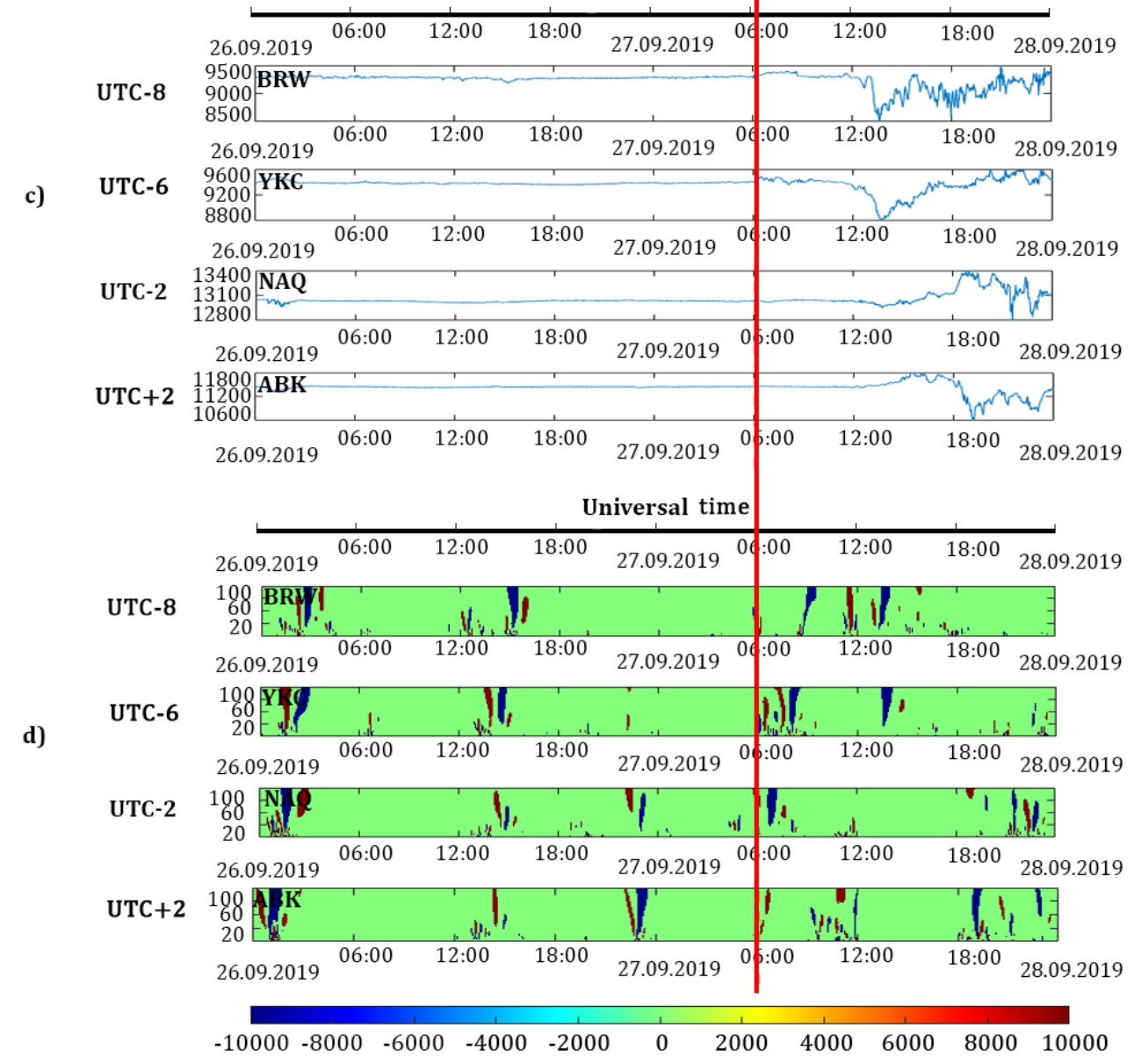

Fig. 9. Results of processing data from auroral stations for the period from 26.09.2019 to 28.09.2019: a) Kp-index; b) Bz-component of IMF, positive values are shown in red, negative values are shown in blue; c) H-components of the geomagnetic field of auroral stations; d) positive (red) and negative (blue) disturbances at auroral stations (operation (3), the size of the sliding time window is 720 samples). The vertical line indicates the beginning of the magnetic storm. 
In the morning hours of September 27, at about 03:00 UT, when the Bz-component IMF turned southward (fig.9b), short-period geomagnetic disturbances are observed at the NAQ auroral station (fig.9d). With the arrival of a high-speed stream, the solar wind speed gradually increased from $375 \mathrm{~km} / \mathrm{s}$ to $500 \mathrm{~km} / \mathrm{s}$, fluctuations of the southern IMF component increased to $\pm 10 \mathrm{nT}$. During the period of increasing oscillations of the Bz-component (fig. $8 \mathrm{~b}$ ), an increase in geomagnetic disturbances is observed at the meridionally located stations (fig. 8d, e), the intensity of which has reached maximum values at the YAK station (fig. 8e). The results of processing data from auroral stations (see fig. 7.9) show a correlation between increases in geomagnetic activity at stations, mainly in the morning and at night, and fluctuations in the southern IMF component (fig. 7d and 9d).

In this work, we performed a cross-correlation analysis of data during the periods of geomagnetic storms on October 2, 2013 and September 27, 2019 to assess the relationship between the identified geomagnetic disturbances and the data of the interplanetary medium (IMF Bz-component and solar wind speed). It is known that the response time of the magnetosphere to fluctuations in the solar wind and magnetic field can vary from several minutes to several days, depending on what physical processes occur in the Earth's magnetosphere. For example, the time delay between the ingress of solar wind energy and the release of energy in the tail of the magnetosphere during a substorm can be 30-60 min [13]. The response time to geomagnetic storms is even longer, since it takes much longer for particles to enter the ring current region [14]. The cross-correlation procedure allows you to detect and study such relationships between the processes under study.

Cross-correlating two datasets involves calculating a Pearson correlation coefficient $r_{k}$ by shifting one dataset in time relative to the other. In the study, the data of the interplanetary medium were stationary, and the values of the H-component of the geomagnetic field and the magnitude $I_{b}$ (see relation (2)) slid over them. Variables were analyzed with hourly and thirty-minute resolution data. The data of the required resolution were obtained by determining the swing of variation for a specific time interval, this made it possible to estimate the rate of change of the analyzed feature, as well as to compensate for gaps in the data. Cross-correlation $r_{k}$ was defined as:

$$
r_{k}=\frac{\sum_{i=1}^{n-k}\left(X_{i}-\bar{X}\right)\left(Y_{i+k}-\bar{Y}\right)}{\sqrt{\sum_{i=1}^{n}\left(X_{i}-\bar{X}\right)^{2} \sum_{i=1}^{n}\left(Y_{i}-\bar{Y}\right)^{2}}},
$$

where $\bar{X}$ and $\bar{Y}$ are the average values of the corresponding series, $k$ is the time lag, which can be positive or negative.

Student's t-test was used to assess the significance of the obtained peak correlation coefficients at $\alpha=0,05$ :

$$
t_{\text {empirical }}=\frac{r \sqrt{n-2}}{\sqrt{1-(r)^{2}}}
$$

where $r$ is the correlation coefficient; $n$ is sample size.

According to the table of critical points of the Student's distribution and according to the chosen level of significance $\alpha$ and the number of degrees of freedom $p=n-2$, critical points were found $t_{\text {critical }}(\alpha ; p)$. If, $\left|t_{\text {empirical }}\right|>t_{\text {critical }}$ then the null hypothesis at the significance level $\alpha$ is turn, i.e. the correlation coefficient is statistically significant; if, 
$\left|t_{\text {empirical }}\right|<t_{\text {critical }}$ then the null hypothesis at the significance level $\alpha$ is accepted, i.e. the correlation coefficient is statistically insignificant.

The values of the obtained peak correlation coefficients and the results of assessing their statistical significance are presented in tables 2-9. Analysis of these results indicates a strong influence of the parameters of the interplanetary medium on the state of the Earth's magnetic field during periods of geomagnetic storms. The results in tables 2-5 show that during the geomagnetic storm on October 02, 2013, in accordance with the significance level $\alpha=0,05$ , 79\% percent of the peak correlation coefficients (highlighted in black in tables 4, 5) are more significant than the correlation coefficients from the tables 2, 3. The results in Tables 6-9 show that during the geomagnetic storm on September 27, 2019, according to the significance level, $57 \%$ of the peak correlation coefficients (highlighted in black in tables 8 , 9) are more significant than the correlation coefficients from the tables 6,7 . Note that the peak correlation coefficients of the GUA station (see table 6, highlighted in black) at a given confidence level of 0,95 are statistically insignificant. The results obtained indicate the consistency and efficiency of the value $I_{b}$.

Figures 10 - 13 show the estimated values of the cross-correlation function during the periods of geomagnetic storms on October 2, 2013 and September 27, 2019 for the Paratunka observatory. The obtained correlograms show that at the Paratunka station a significant influence on the development of the geomagnetic storm of October 2, 2013 during the initial phase was exerted by the magnitude of the Bz-component IMF swing (see fig. 10, $11 \mathrm{a}, \mathrm{c}$ ), and during the main phase - solar wind speed (see fig. 10,11 b, d). During the event of September 27, 2019, the parameters of the interplanetary medium had a significant effect on the development of the magnetic storm during its initial phase, as the storm developed, the dependence gradually decreased, after seven hours the correlation coefficients decreased to 0,2 and lost their statistical significance (fig. 12, 13). It should also be noted that the correlograms obtained from the data of the H-component of the geomagnetic field (the magnitude of the swing) and from the data on the intensity of geomagnetic disturbances should be noted. This result indicates the consistency of the value and the validity of its use to assess the degree of disturbance of the geomagnetic field. It should also be noted that the correlograms obtained from the data of the H-component of the geomagnetic field (swing value) and from the data on the intensity of geomagnetic disturbances $I_{b}$. This result indicates the consistency of the $I_{b}$ value and the validity of its use to assess the degree of disturbance of the geomagnetic field.

Table 2. Results of cross-correlation assessment of the data of the interplanetary medium and the $\mathrm{H}$ component of the geomagnetic field for the period of the geomagnetic storm on October 02, 2013 (hourly resolution)

\begin{tabular}{|l|c|c|c|c|c|c|c|}
\hline $\begin{array}{l}\text { Interp- } \\
\text { lanetary } \\
\text { environment } \\
\text { parameters }\end{array}$ & PET & YAK & GUA & NAQ & YKC & ABK & BRW \\
\hline Bz-component of IMF & $\begin{array}{c}r_{k}=0,76 \\
k=1\end{array}$ & $\begin{array}{c}r_{k}=0,72 \\
k=-2\end{array}$ & $\begin{array}{c}r_{k}=0,68 \\
k=0\end{array}$ & $\begin{array}{c}r_{k}=0,68 \\
k=1\end{array}$ & $\begin{array}{c}r_{k}=0,78 \\
k=1\end{array}$ & $\begin{array}{c}r_{k}=0,70 \\
k=-1\end{array}$ & $\begin{array}{c}r_{k}=0,59 \\
k=-2\end{array}$ \\
\hline Solar wind speed & $\begin{aligned} r_{k}=-0,75 \\
k=4\end{aligned}$ & $\begin{array}{c}r_{k}=-0,63 \\
k=2\end{array}$ & $\begin{array}{c}r_{k}=-0,68 \\
k=4\end{array}$ & $\begin{array}{c}r_{k}=-0,72 \\
k=5\end{array}$ & $\begin{array}{c}r_{k}=-0,67 \\
k=5\end{array}$ & $\begin{array}{c}r_{k}=-0,62 \\
k=3\end{array}$ & $\begin{array}{c}r_{k}=-0,58 \\
k=4\end{array}$ \\
\hline$t_{\text {empirical }}^{\text {Bz }}$ & 5,48 & 4,87 & 4,35 & 4,35 & 5,85 & 4,60 & 3,43 \\
\hline$t^{\text {Solar }}$ empirical & 5,32 & 3,81 & 4,35 & 4,87 & 4,23 & 3,71 & 3,34 \\
\hline
\end{tabular}


Table 3. Results of cross-correlation assessment of the data of the interplanetary medium and the Hcomponent of the geomagnetic field for the period of the geomagnetic storm on October 2, 2013

(thirty-minute resolution)

\begin{tabular}{|l|c|c|c|c|c|c|c|}
\hline $\begin{array}{l}\text { Interp- } \\
\text { lanetary } \\
\text { environment } \\
\text { parameters }\end{array}$ & PET & YAK & GUA & NAQ & YKC & ABK & BRW \\
\hline Bz-component of IMF & $\begin{array}{c}r_{k}=0,74 \\
k=2\end{array}$ & $\begin{array}{c}r_{k}=0,61 \\
k=-2\end{array}$ & $\begin{array}{c}r_{k}=0,62 \\
k=-4\end{array}$ & $\begin{array}{c}r_{k}=0,60 \\
k=-2\end{array}$ & $\begin{array}{c}r_{k}=0,62 \\
k=2\end{array}$ & $\begin{array}{c}r_{k}=0,61 \\
k=-1\end{array}$ & $\begin{array}{c}r_{k}=0,50 \\
k=-3\end{array}$ \\
\hline Solar wind speed & $\begin{array}{c}r_{k}=-0,70 \\
k=7\end{array}$ & $\begin{array}{c}r_{k}=-0,65 \\
k=3\end{array}$ & $\begin{array}{c}r_{k}=-0,64 \\
k=8\end{array}$ & $\begin{array}{c}r_{k}=-0,63 \\
k=10\end{array}$ & $\begin{array}{c}r_{k}=-0,57 \\
k=9\end{array}$ & $\begin{array}{c}r_{k}=-0,61 \\
k=7\end{array}$ & $\begin{array}{c}r_{k}=-0,54 \\
k=4\end{array}$ \\
\hline$t_{\text {empirical }}^{\text {Bz }}$ & 7,46 & 5,22 & 5,36 & 5,09 & 5,36 & 5,22 & 3,92 \\
\hline$t^{\text {Solar }}$ empirical & 6,65 & 5,80 & 5,65 & 5,50 & 4,71 & 5,22 & 4,35 \\
\hline
\end{tabular}

Table 4. Results of cross-correlation assessment of the data of the interplanetary medium and the value of $I_{b}$ for the period of the geomagnetic storm on October 2, 2013 (hourly resolution)

\begin{tabular}{|l|c|c|c|c|c|c|c|}
\hline $\begin{array}{l}\text { Interp- } \\
\text { lanetary } \\
\text { environment } \\
\text { parameters }\end{array}$ & PET & YAK & GUA & NAQ & YKC & ABK & BRW \\
\hline Bz-component of IMF & $\begin{array}{c}r_{k}=0,90 \\
k=1\end{array}$ & $\begin{array}{c}r_{k}=0,63 \\
k=-2\end{array}$ & $\begin{array}{c}r_{k}=0,85 \\
k=-1\end{array}$ & $\begin{array}{c}r_{k}=0,80 \\
k=0\end{array}$ & $\begin{array}{c}r_{k}=0,81 \\
k=1\end{array}$ & $\begin{array}{c}r_{k}=0,70 \\
k=0\end{array}$ & $\begin{array}{c}r_{k}=0,59 \\
k=0\end{array}$ \\
\hline Solar wind speed & $\begin{array}{r}r_{k}=-0,82 \\
k=4\end{array}$ & $\begin{array}{c}r_{k}=-0,81 \\
k=2\end{array}$ & $\begin{array}{c}r_{k}=-0,65 \\
k=3\end{array}$ & $\begin{array}{c}r_{k}=-0,60 \\
k=3\end{array}$ & $\begin{array}{c}r_{k}=-0,69 \\
k=4\end{array}$ & $\begin{array}{c}r_{k}=-0,67 \\
k=3\end{array}$ & $\begin{array}{c}r_{k}=-0,70 \\
k=4\end{array}$ \\
\hline$t^{B z}{ }_{\text {empirical }}^{\text {IAGA code }}$ & $\mathbf{9 , 6 8}$ & 3,81 & $\mathbf{7 , 5 7}$ & $\mathbf{6 , 2 5}$ & $\mathbf{6 , 4 8}$ & $\mathbf{4 , 6 0}$ & $\mathbf{3 , 4 3}$ \\
\hline$t_{\text {empirical }}^{\text {Solar }}$ & $\mathbf{6 , 7 2}$ & $\mathbf{6 , 4 8}$ & 4,01 & 3,52 & $\mathbf{4 , 4 7}$ & $\mathbf{4 , 2 3}$ & $\mathbf{4 , 6 0}$ \\
\hline
\end{tabular}

Table 5. Results of cross-correlation assessment of the data of the interplanetary medium and the value of $I_{b}$ for the period of the geomagnetic storm on October 2, 2013 (thirty-minute resolution)

\begin{tabular}{|c|c|c|c|c|c|c|c|}
\hline $\begin{array}{l}\text { Interp- } \\
\text { lanetary } \\
\text { environment } \\
\text { parameters }\end{array}$ & PET & YAK & GUA & NAQ & YKC & $\mathrm{ABK}$ & BRW \\
\hline Bz-component of IMF & $\begin{array}{c}r_{k}=0,73 \\
k=3\end{array}$ & $\begin{array}{c}r_{k}=0,56 \\
k=-2\end{array}$ & $\begin{array}{c}r_{k}=0,81 \\
k=-1\end{array}$ & $\begin{array}{c}r_{k}=0,74 \\
k=-2\end{array}$ & $\begin{array}{c}r_{k}=0,73 \\
k=2\end{array}$ & $\begin{array}{c}r_{k}=0,63 \\
k=0\end{array}$ & $\begin{array}{c}r_{k}=0,51 \\
k=0\end{array}$ \\
\hline Solar wind speed & $\begin{array}{c}r_{k}=-0,86 \\
k=9\end{array}$ & $\begin{array}{c}r_{k}=-0,65 \\
k=4\end{array}$ & $\begin{array}{c}r_{k}=-0,71 \\
k=6\end{array}$ & $\begin{array}{c}r_{k}=-0,62 \\
k=9\end{array}$ & $\begin{array}{c}r_{k}=-0,65 \\
k=8\end{array}$ & $\begin{array}{c}r_{k}=-0,65 \\
k=8\end{array}$ & $\begin{array}{c}r_{k}=-0,59 \\
k=8\end{array}$ \\
\hline$t_{\text {empirical }}^{B z}$ & 7,24 & 4,58 & 9,37 & 7,46 & 7,24 & $\mathbf{5 , 5 0}$ & 4,02 \\
\hline$t^{\text {Solar }}$ empirical & 11,43 & 5,80 & 6,84 & 5,36 & 5,80 & 5,80 & 4,96 \\
\hline
\end{tabular}


Table 6. Results of cross-correlation assessment of the data of the interplanetary medium and the Hcomponent of the geomagnetic field for the period of the geomagnetic storm on September 27, 2017 (hourly resolution)

\begin{tabular}{|l|c|c|c|c|c|c|c|}
\hline $\begin{array}{l}\text { Interp- } \\
\text { lanetary } \\
\text { environment } \\
\text { parameters }\end{array}$ & PET & YAK & GUA & NAQ & YKC & ABK & BRW \\
\hline Bz-component of IMF & $\begin{array}{c}r_{k}=0,83 \\
k=2\end{array}$ & $\begin{array}{c}r_{k}=0,64 \\
k=-1\end{array}$ & $\begin{array}{c}r_{k}=0,39 \\
k=1\end{array}$ & $\begin{array}{c}r_{k}=0,80 \\
k=2\end{array}$ & $\begin{array}{c}r_{k}=0,61 \\
k=3\end{array}$ & $\begin{array}{c}r_{k}=0,70 \\
k=-1\end{array}$ & $\begin{array}{c}r_{k}=0,64 \\
k=3\end{array}$ \\
\hline Solar wind speed & $\begin{array}{c}r_{k}=0,82 \\
k=0\end{array}$ & $\begin{array}{c}r_{k}=0,85 \\
k=0\end{array}$ & $\begin{array}{c}r_{k}=0,28 \\
k=-2\end{array}$ & $\begin{array}{c}r_{k}=0,79 \\
k=0\end{array}$ & $\begin{array}{c}r_{k}=0,73 \\
k=0\end{array}$ & $\begin{array}{c}r_{k}=0,81 \\
k=0\end{array}$ & $\begin{array}{c}r_{k}=0,82 \\
k=0\end{array}$ \\
\hline$t_{\text {empirical }}^{B z}$ & 6,98 & 3,91 & $\mathbf{1 , 9 9}$ & 6,25 & 3,61 & 4,60 & 3,91 \\
\hline$t_{\text {empirical }}^{\text {Solar }}$ & 6,72 & 7,57 & $\mathbf{1 , 3 7}$ & 6,04 & 5,01 & 6,48 & 6,72 \\
\hline
\end{tabular}

Table 7. Results of cross-correlation assessment of the data of the interplanetary medium and the Hcomponent of the geomagnetic field for the period of the geomagnetic storm on September 27, 2017 (thirty-minute resolution)

\begin{tabular}{|l|c|c|c|c|c|c|c|}
\hline $\begin{array}{l}\text { Interp- } \\
\text { lanetary } \\
\text { environment } \\
\text { parameters }\end{array}$ & PET & YAK & GUA & NAQ & YKC & ABK & BRW \\
\hline Bz-component of IMF & $\begin{array}{c}r_{k}=0,72 \\
k=4\end{array}$ & $\begin{array}{c}r_{k}=0,63 \\
k=-1\end{array}$ & $\begin{array}{c}r_{k}=0,41 \\
k=3\end{array}$ & $\begin{array}{c}r_{k}=0,75 \\
k=5\end{array}$ & $\begin{array}{c}r_{k}=0,51 \\
k=3\end{array}$ & $\begin{array}{c}r_{k}=0,68 \\
k=-1\end{array}$ & $\begin{array}{c}r_{k}=0,57 \\
k=3\end{array}$ \\
\hline Solar wind speed & $\begin{array}{c}r_{k}=0,76 \\
k=-1\end{array}$ & $\begin{array}{c}r_{k}=0,75 \\
k=-1\end{array}$ & $\begin{array}{c}r_{k}=0,41 \\
k=-4\end{array}$ & $\begin{array}{c}r_{k}=0,73 \\
k=-1\end{array}$ & $\begin{array}{c}r_{k}=0,69 \\
k=-1\end{array}$ & $\begin{array}{c}r_{k}=0,79 \\
k=-1\end{array}$ & $\begin{array}{c}r_{k}=0,79 \\
k=0\end{array}$ \\
\hline$t_{\text {empirical }}^{\text {Bz }}$ & 7,04 & 5,50 & 3,05 & 7,69 & 4,02 & 6,29 & 4,71 \\
\hline$t_{\text {Solampirical }}$ & 7,93 & 7,69 & 3,05 & 7,24 & 6,47 & 8,74 & 8,74 \\
\hline
\end{tabular}

Table 8. Results of cross-correlation assessment of the data of the interplanetary medium and the value of $I_{b}$ for the period of the geomagnetic storm on September 27, 2017 (hourly resolution)

\begin{tabular}{|l|c|c|c|c|c|c|c|}
\hline $\begin{array}{l}\text { Interp- } \\
\text { lanetary } \\
\text { environment } \\
\text { parameters }\end{array}$ & PET & YAK & GUA & NAQ & YKC & ABK & BRW \\
\hline Bz-component of IMF & $\begin{array}{c}r_{k}=0,79 \\
k=1\end{array}$ & $\begin{array}{c}r_{k}=0,72 \\
k=1\end{array}$ & $\begin{array}{c}r_{k}=0,61 \\
k=2\end{array}$ & $\begin{array}{c}r_{k}=0,76 \\
k=2\end{array}$ & $\begin{array}{c}r_{k}=0,61 \\
k=2\end{array}$ & $\begin{array}{c}r_{k}=0,70 \\
k=0\end{array}$ & $\begin{array}{c}r_{k}=0,55 \\
k=2\end{array}$ \\
\hline Solar wind speed & $\begin{aligned} r_{k}=0,81 \\
k=-1\end{aligned}$ & $\begin{array}{c}r_{k}=0,91 \\
k=-1\end{array}$ & $\begin{array}{c}r_{k}=0,68 \\
k=-1\end{array}$ & $\begin{array}{c}r_{k}=0,85 \\
k=0\end{array}$ & $\begin{array}{c}r_{k}=0,64 \\
k=-1\end{array}$ & $\begin{array}{c}r_{k}=0,81 \\
k=0\end{array}$ & $\begin{array}{c}r_{k}=0,72 \\
k=-1\end{array}$ \\
\hline$t_{\text {empirical }}^{B z}$ & 6,04 & $\mathbf{4 , 8 7}$ & $\mathbf{3 , 6 1}$ & 5,48 & $\mathbf{3 , 6 1}$ & $\mathbf{4 , 6 0}$ & 3,09 \\
\hline$t_{\text {Solarirical }}^{\text {Sol }}$ & 6,48 & $\mathbf{1 0 , 2 9}$ & $\mathbf{4 , 3 5}$ & $\mathbf{7 , 5 7}$ & 3,91 & $\mathbf{6 , 4 8}$ & 4,87 \\
\hline
\end{tabular}


Table 9. Results of cross-correlation assessment of the data of the interplanetary medium and the value of $I_{b}$ for the period of the geomagnetic storm on September 27, 2017 (thirty-minute resolution)

\begin{tabular}{|l|c|c|c|c|c|c|c|}
\hline $\begin{array}{l}\text { Interp- } \\
\text { lanetary } \\
\text { environment } \\
\text { parameters }\end{array}$ & PET & YAK & GUA & NAQ & YKC & ABK & BRW \\
\hline Bz-component of IMF & $\begin{array}{c}r_{k}=0,70 \\
k=3\end{array}$ & $\begin{array}{c}r_{k}=0,67 \\
k=4\end{array}$ & $\begin{array}{c}r_{k}=0,53 \\
k=4\end{array}$ & $\begin{array}{c}r_{k}=0,70 \\
k=5\end{array}$ & $\begin{array}{c}r_{k}=0,55 \\
k=-11\end{array}$ & $\begin{array}{c}r_{k}=0,67 \\
k=0\end{array}$ & $\begin{array}{c}r_{k}=0,55 \\
k=4\end{array}$ \\
\hline Solar wind speed & $\begin{array}{c}r_{k}=0,80 \\
k=-2\end{array}$ & $\begin{array}{c}r_{k}=0,88 \\
k=-2\end{array}$ & $\begin{array}{c}r_{k}=0,64 \\
k=-1\end{array}$ & $\begin{array}{c}r_{k}=0,82 \\
k=0\end{array}$ & $\begin{array}{c}r_{k}=0,64 \\
k=-1\end{array}$ & $\begin{array}{c}r_{k}=0,80 \\
k=0\end{array}$ & $\begin{array}{c}r_{k}=0,70 \\
k=-2\end{array}$ \\
\hline$t_{\text {empirical }}^{\text {Bz }}$ & 6,65 & $\mathbf{6 , 1 2}$ & $\mathbf{4 , 2 4}$ & 6,65 & $\mathbf{4 , 4 7}$ & 6,12 & 4,47 \\
\hline$t_{\text {empirical }}^{\text {Solar }}$ & $\mathbf{9 , 0 4}$ & $\mathbf{1 2 , 5 7}$ & $\mathbf{5 , 6 5}$ & $\mathbf{9 , 7 2}$ & 5,65 & $\mathbf{9 , 0 4}$ & 6,65 \\
\hline
\end{tabular}

a)

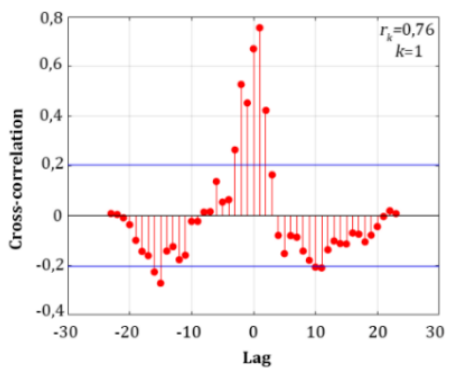

c)

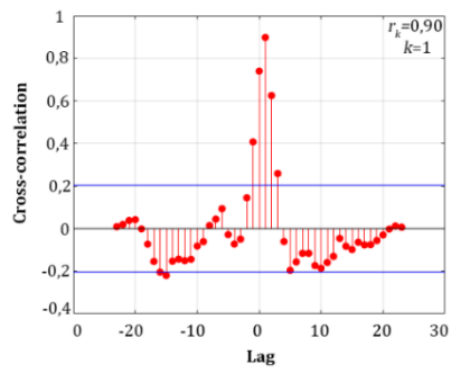

b)
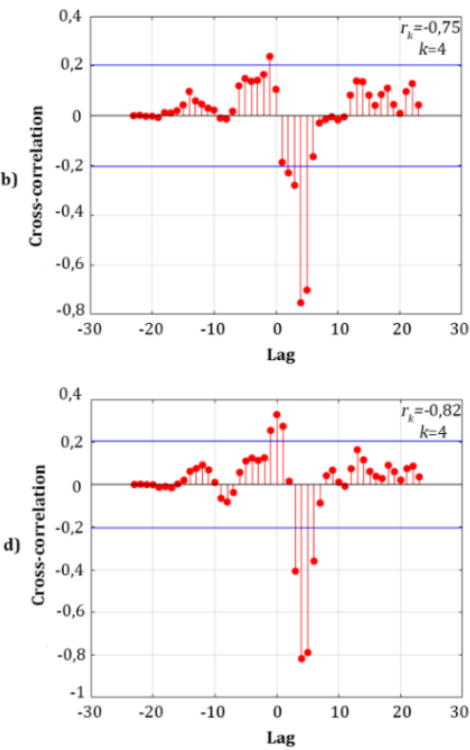

Fig. 10. Correlograms of the «Paratunka» observatory for the period of the geomagnetic storm of 02.10.2013 (hourly resolution): a) correlogram of the H-component of the geomagnetic field and the Bz-component of the IMF; b) correlogram of the H-component of the geomagnetic field and the solar wind speed; c) correlogram of the value $I_{b}$ and Bz-component of the IMF; d) correlogram of the value $I_{b}$ and solar wind speed. 
a)

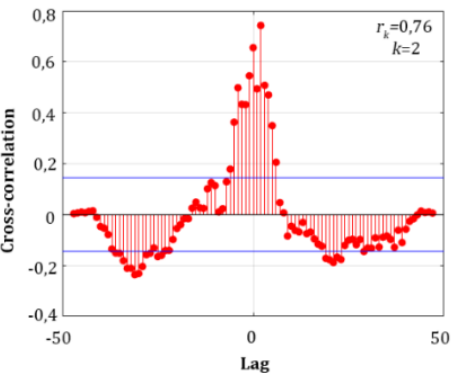

c)

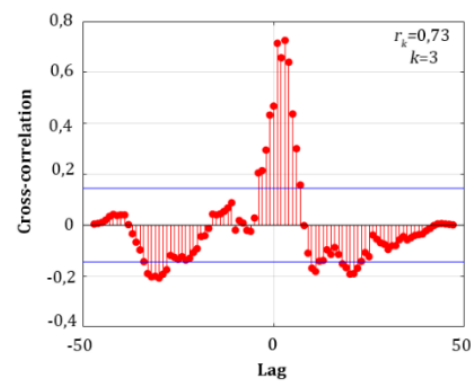

b)
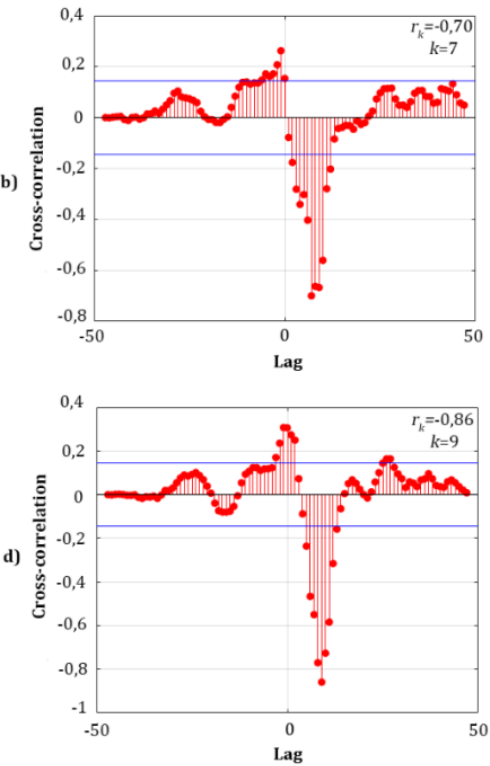

Fig. 11. Correlograms of the «Paratunka» observatory for the period of the geomagnetic storm of 02.10.2013 (thirty minute resolution): a) correlogram of the H-component of the geomagnetic field and the Bz-component of the IMF; b) correlogram of the H-component of the geomagnetic field and the solar wind speed; c) correlogram of the value $I_{b}$ and Bz-component of the IMF; d) correlogram of the value $I_{b}$ and solar wind speed.

a)

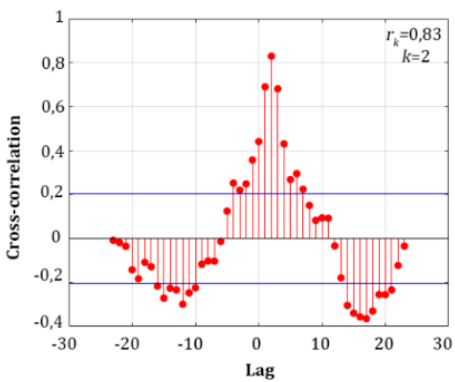

c)

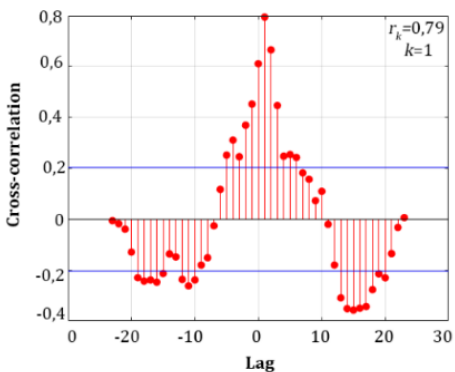

b)
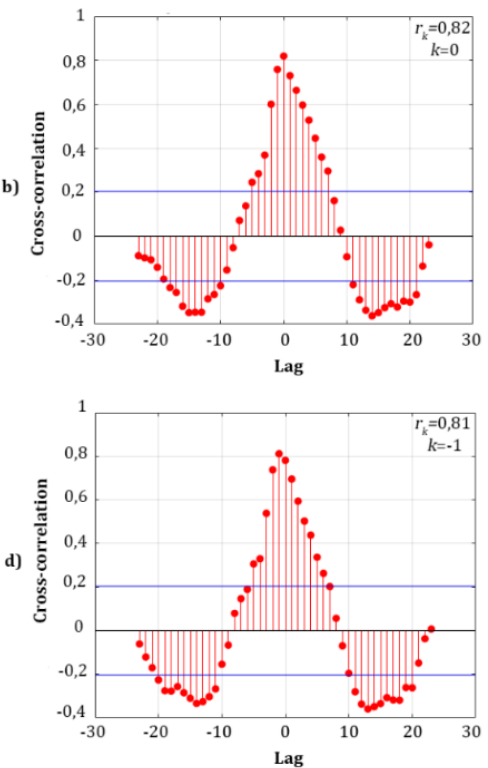

Fig. 12. Correlograms of the «Paratunka» observatory for the period of the geomagnetic storm of 27.09.2019 (hourly resolution): a) correlogram of the H-component of the geomagnetic field and the Bz-component of the IMF; b) correlogram of the H-component of the geomagnetic field and the solar wind speed; c) correlogram of the value $I_{b}$ and Bz-component of the IMF; d) correlogram of the value $I_{b}$ and solar wind speed. 

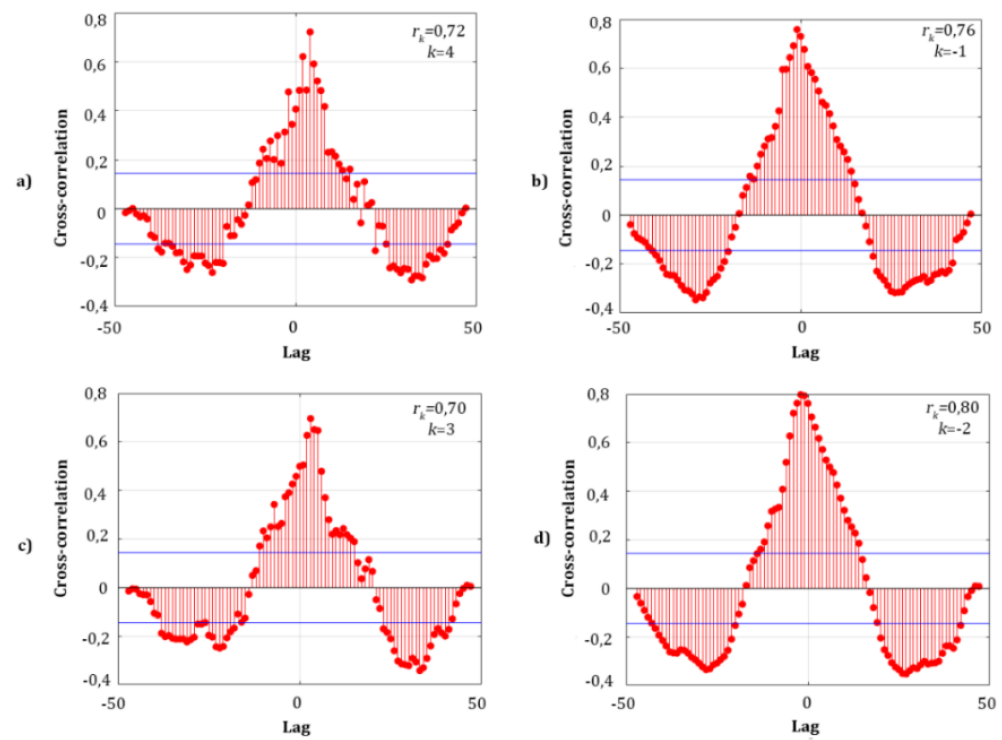

Fig. 13. Correlograms of the «Paratunka» observatory for the period of the geomagnetic storm of 27.09.2019 (thirty minute resolution): a) correlogram of the H-component of the geomagnetic field and the Bz-component of the IMF; b) correlogram of the H-component of the geomagnetic field and the solar wind speed; c) correlogram of the value $I_{b}$ and Bz-component of the IMF; d) correlogram of the value $I_{b}$ and solar wind speed.

\section{Conclusions}

Based on the proposed method, a detailed spatial and temporal analysis of the dynamics of geomagnetic disturbances during the periods of geomagnetic storms was performed on October 02, 2013. (period of high Solar activity) and September 27, 2019 (period of low Solar activity). To evaluate the method, the cross-correlation dependence of the proposed measure of the intensity of geomagnetic disturbances $I_{b}$ and the magnitude of the amplitude of the H-component of the geomagnetic field of the hour and thirty-minute resolutions on the parameters of the interplanetary medium was studied. Based on the results of the study, the following conclusions can be drawn:

1. The peak correlation coefficients obtained during the evaluation of the relationship between the parameters of the interplanetary environment and the intensity of geomagnetic disturbances $I_{b}$ during the periods of geomagnetic storms on October 02, 2013 and September 27, 2019 are more significant in 79\% and 57\% of cases, respectively. The result indicates the consistency and efficiency of the $I_{b}$ value.

2. The observed differences in the correlation profiles are possibly related to the sources of the occurrence of geomagnetic storms (the event on October 2, 2013 was caused by CME, the event on September 27, 2019 was caused by a high-speed flow). On average, the range of the Bz-component IMF had higher peak correlation coefficients for the geomagnetic storm on October 2, 2013, and during the geomagnetic storm on September 27, 2019 , the solar wind velocity data correlated more strongly.

3. According to the correlograms of the «Paratunka» station, a significant influence on the development of the geomagnetic storm of October 2, 2013 during the initial phase was exerted by the magnitude of the Bz-component IMF swing, and during the main phase, by 
the solar wind speed. During the event of September 27, 2019, the parameters of the interplanetary medium had the most significant effect on the development of a magnetic storm during its initial phase.

The authors plan to continue research in this direction, expanding the range of analyzed data and increasing statistics.

The study was carried out within the framework of the state assignment on the topic «Dynamics of physical processes in active zones of near space and geospheres» (2018-2020) № state. registration AAAA-A17-117080110043-4. The authors thank the institutes that support the datalogging stations that were used in the study and thank the developers of INTERMAGNET resources (www.intermagnet.org) and OMNIWebPlus (https://omniweb.gsfc.nasa.gov/hw.html).

\section{References}

1. G. Consolini, R. De Marco, P.De,Michelis, Nonlin. Processes Geophys. 20, 455-466 (2013)

2. A.G. Hafez, T.A. Khan and T.Kohda, Digital Signal Processing20, 715-723 (2010)

3. A. Jach, P. Kokoszka, J. Sojka, L. Zhu, J. Geophys. Res.111, 1-11 (2006)

4. V. Klausner, A.R.R. Papa, O. Mendes, M.O Domingues, P. Frick, Journal of Atmospheric and Solar Terrestrial Physics92, 124-136 (2013)

5. O.V. Mandrikova, I.S. Solovev, T.L. Zalyaev, EPS66 (2014)

6. O.V. Mandrikova, I.S. Solovev, S.Yu. Khomutov, V.V. Geppener, D.M. Klionskiy, M.I. Bogachev, Ann. Geophys.36 1207-1225 (2018)

7. P. Kumar, E. Foufoula-Georgiou, Reviews of Geophysics 35. (1991)

8. A.G. Hafez, E. Ghamry, H. Yayama, K. Yumoto, IEEETGRS50, 4503-4512 (2012)

9. M. Nose, T. Iyemori, M. Takeda, T. Kamei, D.K. Milling, D. Orr, H.J. Singer, E.W. Worthington, Earth Planets Space 50, 773-783 (1998)

10. M. Nose, Earth Planets Space 51, 23-32 (1999)

11. R.L. Bailey, R. Leonhardt, Earth, Planets and Space68 (2016)

12. O.V. Mandrikova, A.I. Rodomanskay, B.S. Mandrikova, Journal of Physics1368 (2019)

13. P.T. Newell, T. Sotirelis, K. Liou, C.-I. Meng, F.J. Rich, Journal of Geophysical Research112 (2007)

14. R. Maggiolo, M. Hamrin, J. De Keyser, T. Pitkänen, G. Cessateur, H. Gunell, L. Maes, Journal of Geophysical Research 122 (2017)

15. I. Daubechies, Ten Lectures on Wavelets (CBMS-NSF Lecture Notes, 1992)

16. C.K. Chui, An introduction to wavelets (Academic Press, New York, 1992)

17. W.D. Gonzalez, B.T. Tsurutani, A.L. Clua-Gonzalez, Space ScienceReviews88 529562 (1999)

18. A.N. Zaitsev, P.A. Dalin, G.N. Zastenker, Geomagnetism and Aeronomy 42, 717-724 (2002) 